POS PROCEEDINGS

\title{
The KLOE-2 detector upgrade at DAФNE
}

\section{Dario Moricciani* on behalf of the KLOE-2 Collaboration}

I.N.F.N. - Sez. Roma "Tor Vergata", Via delle Ricerca Scientifica 1, I-00133 Rome, Italy

E-mail: Dario.Moricciani@roma2.infn.it

In the next few months the KLOE-2 detector is expected to start data taking at the upgraded DA $\phi N E \phi$-factory of INFN Laboratori Nazionali di Frascati. It aims to collect $20 \mathrm{fb}^{-1}$ at the $\phi(1020)$ peak. We review the status of the upgrades on the detector system.

The 2011 Europhysics Conference on High Energy Physics, EPS-HEP 2011,

July 21-27, 2011

Grenoble, Rhône-Alpes, France

\footnotetext{
* Speaker.
} 


\section{Introduction}

The upgrade of the DAФNE machine layout, where the new "Crab-Waist" interaction scheme is implemented [1], offer the possibility to increase the luminosity of a factor $\sim 3$ with respect to the previous data taking campaign and reach a luminosity of the level of $5 \times 10^{32} \mathrm{~cm}^{-2} \mathrm{sec}^{-1}$. This opportunity opens the possibility to collect integrated $20 \mathrm{fb}^{-1}$ in the next three years. The physics program that will be carried on is described in [2]. In order to benefit as much as possible from the luminosity upgrade, new detector have beed developed to increase the acceptance and the tracking resolution. During a first phase of this upgrade the $\gamma \gamma$ taggers will be installed and a first period of data taking is scheduled in the first half of 2012. In a second phase a new tile calorimeter QCALT will be used to instrument the DAФNE focusing system for the detection of photons coming from decays within the drift volume chamber. A Crystal calorimeter CCALT will cover the low $\theta$ region, to increase acceptance for very forward electrons/photons down to 8 degree. A light-material internal tracker (IT) will be installed in the region between the beam pipe and the drift chamber, in order to improve charged vertex reconstruction and to increase the acceptance for low transverse momentum particles. The installation of the QCAL, CCALT and IT is scheduled for the 2012 summer shutdown and the data taking will start on beginning 2013

\section{2. $\gamma \gamma$ taggers}

In order to have access to the reaction $e^{+} e^{-} \rightarrow e^{+} e^{-} \gamma \gamma^{1}$ in the energy region of the $\phi$ meson production, new detectors along the beam line have to be installed in order to catch the scattered $e^{+} e^{-}$. Our simulation shows that we need to place new detectors in two different regions on both side of the Interaction Point (IP):

- inside the KLOE detector, where we can detect leptons with a energy between 50 and 450 $\mathrm{MeV}$;

- after DAФNE bending dipole, where we detect the final leptons having an energy greater than $420 \mathrm{MeV}$.

Two new detectors, namely Low Energy Tagger (LET) and High Energy Tagger (HET) have been already installed and will be used since the beginning of data taking.

\subsection{LET Detector}

This detector [3] is located inside KLOE, $1.5 \mathrm{~m}$ away form the IP. The environmental conditions require radiation-tolerant devices, insensitive to magnetic field. Moreover this detector has to be small in size and has to provide a good energy resolution for the measurement of the $\gamma \gamma$ invariant mass and a good time resolution to associate the detected events with the proper bunch crossing. The selected choice to fulfill these requirements is a high-Z scintillator with high light yield and fast emission: the new Cerium doped Lutetium Yttrium Orthosilicate (LYSO) crystal scintillators, coupled to silicon photomultipliers (SiPM).

\footnotetext{
${ }^{1}$ And then $\gamma \gamma \rightarrow X$, where $X$ stands for any hadronic state allowed by the conservation law.
} 


\subsection{HET Detector}

The HET detector is a position detector used for measuring the deviation of leptons from their main orbit. By means of this measurement and of its timing, we are able to disentangle, and thus to tag $\gamma \gamma$ physics events. The HET detectors are placed at the exit of the DAФNE dipole magnets, $11 \mathrm{~m}$ away from the IP, both on positron and electron arm. The HET sensitive area is made up of a set of 28 plastic scintillators. The dimensions of each of them are $3 \mathrm{~mm} \times 5 \mathrm{~mm} \times 6 \mathrm{~mm}$. One additional scintillator, with dimensions: $3 \mathrm{~mm} \times 50 \mathrm{~mm} \times 6 \mathrm{~mm}$, is used for coincidence purposes. The light emitted by each of the 28 scintillators is carried out through a plastic light guide. The 28 scintillators are placed at different distances from the beamline, in such a way that the measurement of the distance between the hitting particle and the beam can be performed simply knowing which scintillator has fired. On the vertical plane the scintillators are aligned, exception made for a slight curvature at big distance from the beam. They show their $5 \mathrm{~mm} \times 6 \mathrm{~mm}$ face to the impinging particles that cross them along the $3 \mathrm{~mm}$ thickness. The scintillators are not placed side by side, on the contrary there is an overlap of $0.5 \mathrm{~mm}$ on the $5 \mathrm{~mm}$ side. In the horizontal plane, the scintillators are arranged in a two-staircase fashion. This is done in order to make room for the light guides connecting every scintillator with the outside. Each light guide is connected to the 3 $\mathrm{mm} \times 6 \mathrm{~mm}$ face of a scintillator. Once outside, all the guides are bent and coupled to the light sensors Hamamatsu R9880U-110 SEL photomultipliers which have a high quantum efficiency of $\sim 35 \%$. The time resolution of this system is of the order of $200 \mathrm{ps}$. A dedicated DAQ electronics board based on a Xilinx Virtex-5 FPGA [4] have been developed for this detector. It provides a MultiHit TDC with a time resolution of the order of $\sim 500 \mathrm{ps}$ and the possibility to acquire data any $2.1 \mathrm{nsec}$. These performances are needed in order to cleary identify the right bunch crossing.

\section{QCALT Detector}

Particles coming from secondary vertexes inside the drift chamber volume can hit one of the quadrupole magnets escaping detection. For this reason we already had a calorimeter around the quadrupole in the past: the QCAL detector. The rare decay $K_{L} \rightarrow 2 \pi^{0}$ is an example of the physics channels that benefits of thisdetector since the most important background source in this measurement is $K_{L} \rightarrow 3 \pi^{0}$. The new QCALT [5] calorimeter will provide better performances. The higher detection efficiency and the improved granularity will increase both background rejection and the efficiency for the signal. The QCALT detector will be composed of two tile calorimeters with wavelength shifter and SiPM readout. It will have a dodecagonal structure (1 $\mathrm{m}$ length) made by 5 layers of tungsten $(3.5 \mathrm{~mm})$ tiles $(5 \mathrm{~mm})$ and air gap $(1 \mathrm{~mm})$. Each layer is composed by 16 cells with $5 \mathrm{~cm} \times 5 \mathrm{~cm}$ granularity for a total of $\sim 2000$ readout channels.

\section{CCALT Detector}

In order to increase the angular efficiency of the KLOE electromagnetic calorimeter, an additional small detector will be placed in front of the quadrupole magnets QD0. The present electromagnetic calorimeter covers down to 21 degrees. With CCALT [6] the coverage will go down to 8 degrees. With such a detector we can improve the measurement of the branching ratio of the reaction $K_{S} \rightarrow \gamma \gamma$ together with the measurement of the transition form factor of the $\pi^{0}$, that will 
be performed by means of the $\gamma \gamma$ reaction $e^{+} e^{-} \rightarrow e^{+} e^{-} \pi^{0}$. The major background for the process $K_{S} \rightarrow \gamma \gamma$ is the decay channel $K_{S} \rightarrow \pi^{0} \pi^{0} \rightarrow 4 \gamma$, with two photons lost in the beam pipe or due to the EmC inefficiency. With the CCALT calorimeter we will increase the efficiency on small angle photon detection, thus improving the precision on this measurement. The CCALT will be made of 2 small barrels of 48 LYSO crystals of trapezoidal shape and a length of $8-9 \mathrm{~cm}$ and transversal area of $(1.5 \times 1.5) \mathrm{cm}^{2}$ in the readout face, equipped with a large area SiPM.

\section{Inner Tracker Detector}

An inner tracker (IT) detector [7] will be installed to improve vertex reconstruction. The requirements for this kind of detector are good space resolution: $\sigma_{r \phi} \sim 200 \mu \mathrm{m}$ and $\sigma_{z} \sim 500$ $\mu \mathrm{m}$, low material budget: $<2 \%$ of a radiation length and high rate capability: $5 \mathrm{kHz} / \mathrm{cm}^{2}$. The adopted solution is the Cylindrical-GEM (CGEM), a triple-GEM detector composed by concentric cylindrical electrodes. Each layer consist of a cathode, 3 GEM foils for the multiplication stage (gain $\sim 10^{4}$ ) and an anode, acting also as the readout circuit. The high rate capability of the GEM (up to $1 \mathrm{MHz} / \mathrm{mm}^{2}$ ) makes this detectors suitable to be placed near the interaction point of a highluminosity collider machine. The IT will be then composed by 4 concentric CGEM detection layers at radii from $13 \mathrm{~cm}$, to preserve the $K_{S}-K_{L}$ quantum interference region, to $23 \mathrm{~cm}$ as constrained by the DC inner wall diameter of $25 \mathrm{~cm}$. The total active length for all layers will be $70 \mathrm{~cm}$. The anode readout of each CGEM will be segmented with $650 \mu \mathrm{m}$ pitch XV patterned strips with a stereo angle of $\sim 40^{\circ}$, for a total of about 30000 FEE channels. The Front-End Electronics for the IT is based on the new GASTONE ASIC [8], a 64 channels chip composed by four different stages: a charge preamplifier with $20 \mathrm{mV} / \mathrm{fC}$ sensitivity, a shaper, a leading-edge discriminator with a programmable threshold and a monostable stretcher of the digital signal, to synchronize with the KLOE-2 Level1 trigger.

\section{References}

[1] M. Zobov et al. Phys. Rev.Lett.104 2010174801.

[2] G. Amelino-Camelia et al. Eur. Phys. J. C68 (2010) 619.

[3] D. Babusci at al. Nucl. Instrum. Meth. A617 (2010) 81.

[4] L. Iafolla at al. Nucl. Instrum. Meth. A617 (2010) 272.

[5] M. Cordelli et al., arxiv 0906.1133.

[6] M. Cordelli et al., Jour. of Phys. 293, (2011) 012010.

[7] KLOE-2 Collaboration, arxiv 1002.2572.

[8] A. Balla al. Nucl. Instrum, Meth. 604 (2009) 23. 